\title{
LUT
}

Lappeenranta

University of Technology

\section{Transmit Antenna Selection in Wireless-Powered Communication Networks}

Badarneh Osamah. S., Benevides da Costa Daniel, Nardelli Pedro Henrique Julian

This is a Post-print

version of a publication

published by IEEE

in 2019 IEEE 30th Annual International Symposium on Personal, Indoor and Mobile Radio Communications (PIMRC)

DOI: $\quad$ 10.1109/PIMRC.2019.8904398

Copyright of the original publication: (c) Copyright 2019 IEEE

Please cite the publication as follows:

Badarneh O. S., Benevides da Costa D., Nardelli P. H. J. (2019). Transmit Antenna Selection in Wireless-Powered Communication Networks. Published in 2019 IEEE 30th Annual International Symposium on Personal, Indoor and Mobile Radio Communications (PIMRC), 8-11 Sept. 2019, Istanbul, Turkey. DOI: 10.1109/PIMRC.2019.8904398 


\section{Transmit Antenna Selection in Wireless-Powered Communication Networks}

\author{
Osamah. S. Badarneh \\ School of Electrical Engineering \\ and Information Technology \\ German Jordanian University \\ Email: Osamah.Badarneh@gju.edu.jo
}

\author{
Daniel Benevides da Costa \\ Department of Computer Engineering \\ Federal University of Ceará, Brazil \\ Email: danielbcosta@ieee.org
}

\author{
Pedro Henrique Juliano Nardelli \\ School of Energy Systems \\ LUT, Finland \\ Email: pedro.nardelli@lut.fi
}

\begin{abstract}
In this paper, we consider a wireless-powered communication network with transmit antenna selection (TAS), where an energy-limited multi-antenna information source, powered by a dedicated power beacon, communicates with a mobile user (MU). The MU is equipped with a single-antenna and its mobility is characterized by the well-known random waypoint mobility model. Differently from previous works which considered only static scenarios, this paper aims to investigate wireless power and information transfer in the scenario with a random mobile user under Nakagami- $m$ fading. To this end, exact analytical expressions for the outage probability, average delay-limited throughput, ergodic capacity, and average delay-tolerant throughput are derived. The analytical results are compared with Monte-Carlo simulations in order to validate the analysis and provide useful insights on the impact of different parameters on the system performance.
\end{abstract}

Index Terms-Energy harvesting, multi-antenna, Nakagami- $m$ fading, random way-point mobility.

\section{INTRODUCTION}

Wireless energy transfer (WET) technology offers a new paradigm to prolong the lifetime of energyconstrained wireless systems [1]. In such a technology, wireless devices can harvest energy remotely from the radio-frequency (RF) signals radiated by energy transmitters. Similar to wireless information transmission, WET also suffers from various propagation loss, such as shadowing, path loss, and fading. One important application of RF-enabled WET is wireless powered communications (WPCs). In the literature, there are basically two scenarios of WPCs. In the first scenario, an access point (AP) sends energy signal to users in the downlink mode [2]-[4]. Then, users harvest energy and employ it to send information to the AP in the uplink mode. In the second scenario, wireless devices harvest energy from RF signals emitted from a (dedicated) power transmitter before their information transmission [5]-[8]. In both scenarios, the "harvest-then-transmit" protocol proposed in [1], [9] is implemented.

Over the last few years, the performance of different communication systems in the context of wireless power transfer has been extensively investigated. In [10], the authors investigated capacity performance in WPC system under Rician fading channels. In [11], closed-form expressions of the average throughput for delay-limited and delay-tolerant transmission modes in WPC network over Rayleigh fading were derived. The tradeoff between harvested energy and information rate in a simultaneous wireless information and power transfer (SWIPT) system under time-switching (TS) and power-splitting (PS) schemes was investigated in [12]. The effect of cochannel interference (CCI) in WPC system on the average signal-to-interference-plus-noise ratio, the outage probability and the average throughput were examined in [13]. The performance of average bit error rate (BER) of space-shift-keying-based WPC was analyzed in [14]. The authors in [15] studied the performance of downlink multiuser scheduling for a time-slotted system with SWIPT. A dynamic PS scheme in which the received signal is split into two streams with adjustable power levels for information decoding and energy harvesting separately based on the instantaneous channel condition that is assumed to be known at the receiver was proposed in [16].

A common feature of prior works is that they considered only static scenarios in which the transmitter sends information to a static user. Unlike existing works, this paper aims to analyze the performance of wirelesspowered communication networks (WPCNs) considering a mobile user (MU). Specifically, we consider a WPCN where a multi-antenna access point (AP) transmitter harvests energy from a power beacon (PB) before transmit the data to a mobile MU. It is noteworthy that the considered WPCN setup was widely adopted in the literature [5]-[8]. Additionally, we assume that the receiving node mobility is governed by the random waypoint (RWP) model, which is a commonly used mobility model to evaluate different wireless systems. Based on the considered setup, exact analytical expressions for the outage probability, average delay-limited throughput, ergodic capacity, and average delay-tolerant throughput are derived. The analytical results are compared with Monte-Carlo simulations in order to validate the analysis 


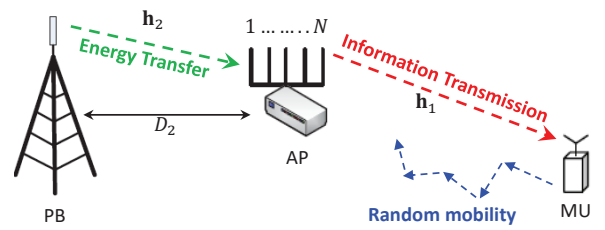

Fig. 1: System model for wireless energy and information transfer.

and provide useful insights on the impact of different parameters on the system performance.

\section{System, Channel, And Mobility Models}

Consider a wireless powered network as shown in Fig. 1, which consists of a $\mathrm{PB}$, transmitter $\mathrm{AP}$, and MU. In this network, $\mathrm{PB}$ and $\mathrm{MU}$ are equipped with a single antenna, while AP is equipped with $N$ antennas. We assume that AP is an energy-constrained node and harvests the RF energy transferred by PB before data transmission to MU. We adopt the time-splitting protocol proposed in [1], [5]-[8]. As such, the total transmission time slot $T$ is divided into two orthogonal sub-slots. The first time slot is dedicated for power transfer from $\mathrm{PB}$ to AP with time duration of $\tau T$, with $\tau(0<\tau<1)$ being the time-splitting ratio. In the second time slot, i.e., $(1-\tau) T$, the AP applies the harvested energy to transmit the information to the MU. Unlike the existing works in the literature, it is assumed that the RWP is used to model the mobility of MU. Also, we assume that all channels are modeled as quasi-static fading and remain unchanged during each time block but vary independently from one block to another.

During the energy harvesting phase, the received signal at AP $\mathbf{y}_{2}$ can be expressed as

$$
\mathbf{y}_{2}=\sqrt{\frac{P_{s}}{D_{2}^{\alpha}}} \mathbf{h}_{2} x_{s}+\mathbf{n}_{2},
$$

where $P_{s}$ is the transmit power of the $\mathrm{PB}, D_{2}$ is the distance between BP and AP, $\alpha$ is the path loss exponent, the $N \times 1$ vector $\mathbf{h}_{2}$ denotes the power transfer channel between PB and AP, $x_{s}$ is the energy signal normalized as $\mathbb{E}\left[\left|x_{s}\right|^{2}\right]=1$, whereby $\mathbb{E}[\cdot]$ and $|\cdot|$ are the expectation operator and the absolute value, respectively. $\mathbf{n}_{2}$ is an $N$-dimensional additive white Gaussian noise (AWGN) vector with $\mathbb{E}\left[\mathbf{n}_{2} \mathbf{n}_{2}^{\dagger}\right]=\sigma^{2} \mathbf{I}_{N}$, where $(\cdot)^{\dagger}$ is the complex conjugate operator. $\sigma^{2}$ is the noise power, and $\mathbf{I}_{N}$ is the identity matrix of size $N$.

At the end of the first phase, the total amount of energy harvested by the AP during the period $\tau T$, denoted by $E_{h}$, is given by

$$
E_{h}=\lambda \tau T \frac{P_{s}\left\|\mathbf{h}_{2}\right\|^{2}}{D_{2}^{\alpha}}
$$

where $\lambda(0<\lambda<1)$ is the energy conversion efficiency and $\|\cdot\|$ denotes the Frobenius norm.
During the second phase with duration $(1-\tau) T$, the transmit power at AP can be computed as

$$
P_{t}=\frac{E_{h}}{(1-\tau) T}=\lambda P_{s}\left\|\mathbf{h}_{2}\right\|^{2} \frac{\tau}{(1-\tau) D_{2}^{\alpha}} .
$$

In this paper, Nakagami- $m$ fading distribution is used to model the power transfer channel, i.e., the amplitude of each element of $\mathbf{h}_{2}$ follows Nakagami- $m$ distribution with shape parameter $m_{2}$ and average power $\Omega_{2}$. Also, the channel between the AP and the MU, $\mathbf{h}_{1}$, follows the Nakagami- $m$ distribution with shape parameter $m_{1}$ and average power $\Omega_{1}$.

Turning now out attention to the RWP mobility model employed to describe the mobility of the MU. The PDF of the distance $r$ between the AP and MU can be written by the general form as [17]

$$
f_{r}(r)=\sum_{\ell=1}^{n} \frac{B_{\ell}}{D^{\beta_{\ell}+1}} r^{\beta_{\ell}}, \quad 0 \leq r \leq D
$$

where $D$ is the maximum distance between $\mathrm{AP}$ and MU. The parameters $n, B_{\ell}$ and $\beta_{\ell}$ depend on the number of dimensions for a given network topology. For 1D, $n=2$, $B_{\ell}=[6,-6]$ and $\beta_{\ell}=[1,2]$, while for $2 \mathrm{D}, n=3$, $B_{\ell}=(12 / 73)[27,-35,8]$, and $\beta_{\ell}=[1,3,5]$, and for 3D, $n=3, B_{\ell}=(35 / 72)[21,-34,13]$, and $\beta_{\ell}=[2,4,6]$. In RWP mobility model, the receiving node is located at randomly selected coordinate points in the service area, which depends on the network topology. For a 1D topology, the transmitter is located at the origin while the receiving node moves on a line. The $2 \mathrm{D}$ topology is assumed to be a circle, whereas a 3D topology is a spherical network. For 2D and 3D topologies, the transmitter is placed at the origin of a circle and sphere, respectively.

\section{A. Transmit Antenna Selection (TAS)}

In TAS, the antenna with the maximum AP-MU channel gain is selected, i.e.,

$$
g=\arg \max _{i=1, \cdots, N}\left|h_{i d}\right|^{2},
$$

where $h_{i d}$ is the $i$-th element of main channel $\mathbf{h}_{1}$. Therefore, the PDF of $g$ can be obtained via

$$
f_{g}(x)=N\left(F_{Z}(x)\right)^{N-1} f_{Z}(x),
$$

where $f_{Z}(x)$ and $F_{Z}(x)$ are, respectively, the PDF and CDF of the Nakagami- $m$ fading channel (AP-MU channel), which are given by

$$
f_{Z}(x)=\frac{1}{\Gamma\left(m_{1}\right)}\left(\frac{m_{1}}{\Omega_{1}}\right)^{m_{1}} x^{m_{1}-1} \exp \left(-\frac{m_{1}}{\Omega_{1}} x\right),
$$

and

$$
F_{Z}(x)=\frac{\gamma\left(m_{1}, \frac{m_{1}}{\Omega_{1}} x\right)}{\Gamma\left(m_{1}\right)},
$$


where $\Gamma(\cdot)$ and $\gamma(\cdot, \cdot)$ are the Gamma and lower incomplete Gamma functions [18, Eq. (8.31)], [18, Eq. (8.350.1)], respectively.

Using (7) and (8), the PDF in (6) can be derived as

$$
\begin{aligned}
f_{g}(x)=\frac{N}{\left[\Gamma\left(m_{1}\right)\right]^{N}} \exp \left(-\frac{m_{1} N}{\Omega_{1}} x\right) \\
\quad \times \sum_{k=0}^{\infty} c_{k}\left(\frac{m_{1}}{\Omega_{1}}\right)^{k+m_{1} N} x^{k+m_{1} N-1},
\end{aligned}
$$

where $c_{0}=\left(\frac{1}{m_{1}}\right)^{N-1}$ and $c_{v}=$ $\frac{\Gamma\left(m_{1}+1\right)}{v} \sum_{k=1}^{v} \frac{k N-v}{\Gamma\left(m_{1}+k+1\right)} c_{v-k}$ for $v \geq 1$.

The PDF of the signal power, at AP, is given by

$$
\begin{aligned}
f_{\left\|\mathbf{h}_{2}\right\|^{2}}(x)=\frac{1}{\Gamma\left(m_{2} N\right)}\left(\frac{m_{2}}{\Omega_{2}}\right)^{m_{2} N} & x^{m_{2} N-1} \\
& \times \exp \left(-\frac{m_{2}}{\Omega_{2}} x\right),
\end{aligned}
$$

By its turn, received power at MU can be expressed as

$$
\Omega_{1}=K_{1}\left\|\mathbf{h}_{2}\right\|^{2} r^{-\alpha},
$$

where $K_{1}=\lambda P_{s} \frac{\tau}{(1-\tau) D_{2}^{\alpha}}$.

Moreover, it can be seen that the conditional PDF of the received signal power at MU can be expressed as

$$
\begin{aligned}
& f_{X}(x \mid r, y)=f_{g}\left(x, \Omega_{1}=K_{1} y r^{-\alpha}\right)=\frac{N}{\left[\Gamma\left(m_{1}\right)\right]^{N}} \\
& \times e^{-\frac{m_{1} N}{K_{1} y r^{-\alpha}} x} \sum_{k=0}^{\infty} c_{k}\left(\frac{m_{1}}{K_{1} y r^{-\alpha}}\right)^{k+m_{1} N} x^{k+m_{1} N-1},
\end{aligned}
$$

where $y=\left\|\mathbf{h}_{2}\right\|^{2}$.

Thus, applying concepts of probability, the unconditional PDF of the received signal power at MU can be given by

$$
f_{X}(x)=\int_{0}^{D} \int_{0}^{\infty} f_{X}(x \mid r, y) f_{Y}(y) f_{r}(r) d y d r .
$$

Now, using (4), (10), and (12), the unconditional PDF of the received signal power at $\mathrm{MU}$ can be written as follows

$$
\begin{aligned}
& f_{X}(x)=\frac{N}{\left[\Gamma\left(m_{1}\right)\right]^{N} \Gamma\left(m_{2} N\right)}\left(\frac{m_{2}}{\Omega_{2}}\right)^{m_{2} N} \sum_{k=0}^{\infty} \sum_{\ell=1}^{n} c_{k} \\
& \frac{B_{\ell}}{D^{\beta_{\ell}+1}}\left(\frac{m_{1}}{K_{1}}\right)^{k+m_{1} N} x^{k+m_{1} N-1} \times \int_{0}^{D} r^{\alpha\left(k+m_{1} N\right)+\beta_{\ell}} \\
& \int_{0}^{\infty} y^{m_{2} N-m_{1} N-k-1} \exp \left(-\frac{m_{1} N x r^{\alpha}}{K_{1} y}-\frac{m_{2}}{\Omega_{2}} y\right) d y d r .
\end{aligned}
$$

The inner integral w.r.t. $y$ can be solved in closed-form using [18, Eq. (3.471.9)]. Thus, (14) reduces to (15), at the top of the next page. The unconditional PDF in (15) can be obtained, by representing the function $K_{v}(\cdot)$ in terms of Meijer's G-function [19, Eq. (14)], using [19, Eq. (26)], [20, Eq. (8.2.2.15)], and after some algebraic manipulations, as in (16), where $\mathrm{G}_{u, v}^{s, t}[\cdot \mid \cdot]$ is the Meijer's G-function [18, Eq. (9.301)]. On the other hand, the corresponding CDF can be obtained as in (17). To the best of authors' knowledge, (16) and (17) are new.

\section{Performance Analysis}

\section{A. Delay-Limited Transmission Mode}

In delay-limited transmission mode, the average throughout can be obtained by evaluating the outage probability with a fixed transmission rate [1]. That is

$$
\rho_{\text {Lim }}=\left(1-P_{\text {out }}\right)(1-\tau) R,
$$

where $R$ is the constant transmission rate, and $P_{\text {out }}$ is the outage probability, which is defined as the probability that the instantaneous channel capacity drops below the source's fixed transmission rate $R$, that is

$$
P_{\text {out }}=\operatorname{Pr}\left(\log _{2}(1+x)<R\right)=\operatorname{Pr}\left(x<x_{0}\right),
$$

where $\operatorname{Pr}\left(x<x_{0}\right)=F_{X}\left(x_{0}\right)$ with $x_{0}=2^{R}-1$ and $F_{X}(\cdot)$ is given in (17).

The average throughout of the delay-limited transmission mode can be obtained using (17), (18), and (19). The result is shown in (20). Note that the coefficients $c_{v} \geq 0$ is for all values of $v$. Thus, it is worthwhile to emphasize that (20) converges rapidly and steadily, requiring few terms (i.e., $\sim 20$ terms) for an error smaller than $10^{-8}$.

\section{B. Delay-Tolerant Transmission Mode}

In delay-tolerant transmission mode, the average throughput can be calculated by evaluating the ergodic capacity at any constant rate [1], i.e.,

$$
\rho_{T o l}=(1-\tau) C,
$$

where $C$ is the ergodic capacity of the system, which can be defined as

$$
C=\frac{1}{\ln (2)} \int_{0}^{\infty} \ln (1+x) f_{X}(x) d x .
$$

Using (16) and (22), then representing the logarithmic function in terms of Meijer's G-function [Eq. (8.4.6.5)], and capitalizing on [20, Eq. (2.24.1.1)], the ergodic capacity can be attained as in (23), at the bottom of the next page.

Accordingly, the average throughput of the delaytolerant transmission mode can be obtained using (21) and (23) as in (24), at the bottom of the next page. Note that (23) and (24) have not been reported in the literature as far as the authors are aware. Moreover, although (23) and (24) are expressed in terms of infinite series, however, the coefficients $c_{v} \geq 0$ for all values of $v$. Therefore, (23) and (24) converge rapidly and steadily, requiring few terms for accurate results. 


$$
\begin{aligned}
f_{X}(x)=\frac{2 N}{\left[\Gamma\left(m_{1}\right)\right]^{N} \Gamma\left(m_{2} N\right)} \sum_{k=0}^{\infty} \sum_{\ell=1}^{n} c_{k} \frac{B_{\ell}\left(\frac{m_{1} m_{2}}{K_{1} \Omega_{2}}\right)^{\frac{1}{2}\left(m_{2} N+m_{1} N+k\right)}}{D^{\beta_{\ell}+1}} N^{\frac{1}{2}\left(m_{2} N-m_{1} N-k\right)} x^{\frac{1}{2}\left(m_{2} N+m_{1} N+k\right)-1} \\
\quad \times \int_{0}^{D} r^{\frac{\alpha}{2}\left(m_{2} N+m_{1} N+k\right)+\beta_{\ell}} K_{m_{2} N-m_{1} N-k}\left(2 \sqrt{\frac{m_{1} m_{2} N x r^{\alpha}}{K_{1} \Omega_{2}}}\right) d r .
\end{aligned}
$$

$$
\begin{aligned}
f_{X}(x)=\frac{N}{\alpha\left[\Gamma\left(m_{1}\right)\right]^{N} \Gamma\left(m_{2} N\right)} \sum_{k=0}^{\infty} \sum_{\ell=1}^{n} & c_{k} \frac{B_{\ell}}{D^{\beta_{\ell}+1}}\left(\frac{K_{1} \Omega_{2}}{m_{1} m_{2} N}\right)^{\frac{\beta_{\ell}+1}{\alpha}}\left(\frac{1}{N}\right)^{k+m_{1} N} x^{-\frac{\beta_{\ell}+1}{\alpha}-1} \\
& \times \mathrm{G}_{1,3}^{2,1}\left[\frac{m_{1} m_{2} N D^{\alpha}}{K_{1} \Omega_{2}} x \mid m_{2} N+\frac{\beta_{\ell}+1}{\alpha}, k+m_{1} N+\frac{\beta_{\ell}+1}{\alpha}, 0\right]
\end{aligned}
$$

$$
\begin{aligned}
F_{X}(x)=\frac{N}{\alpha\left[\Gamma\left(m_{1}\right)\right]^{N} \Gamma\left(m_{2} N\right)} \sum_{k=0}^{\infty} & \sum_{\ell=1}^{n} c_{k} \frac{B_{\ell}}{D^{\beta_{\ell}+1}}\left(\frac{K_{1} \Omega_{2}}{m_{1} m_{2} N}\right)^{\frac{\beta_{\ell}+1}{\alpha}}\left(\frac{1}{N}\right)^{k+m_{1} N} x^{-\frac{\beta_{\ell}+1}{\alpha}} \\
& \times \mathrm{G}_{2,4}^{2,2}\left[\left.\frac{m_{1} m_{2} N D^{\alpha}}{K_{1} \Omega_{2}} x\right|_{\left.m_{2} N+\frac{\beta_{\ell}+1}{\alpha}, k+m_{1} N+\frac{\beta_{\ell}+1}{\alpha}, \frac{\beta_{\ell}+1}{\alpha}, 0\right] .} .\right.
\end{aligned}
$$

$$
\begin{aligned}
\rho_{\text {Lim }}=(1-\tau) R\{1- & \frac{N}{\alpha\left[\Gamma\left(m_{1}\right)\right]^{N} \Gamma\left(m_{2} N\right)} \sum_{k=0}^{\infty} \sum_{\ell=1}^{n} c_{k} \frac{B_{\ell}}{D^{\beta_{\ell}+1}}\left(\frac{K_{1} \Omega_{2}}{m_{1} m_{2} N}\right)^{\frac{\beta_{\ell}+1}{\alpha}}\left(\frac{1}{N}\right)^{k+m_{1} N} x_{0}^{-\frac{\beta_{\ell}+1}{\alpha}} \\
& \left.\times \mathrm{G}_{2,4}^{2,2}\left[\frac{m_{1} m_{2} N D^{\alpha}}{K_{1} \Omega_{2}} x_{0} \mid m_{2} N+\frac{\beta_{\ell}+1}{\alpha}, k+m_{1} N+\frac{\beta_{\ell}+1}{\alpha}, \frac{\beta_{\ell}+1}{\alpha}, 0\right]\right\} .
\end{aligned}
$$

\section{AsYmptotic ANALYSIS}

To obtain further insight into the derived expressions, in the this section, we derive asymptotic analytical expressions for the outage probability. Asymptotic analyses can be carried out based on the behavior of the PDF of the received signal power around the origin. As such, for small values of $x$, the modified Bessel function $K_{v}(x)$ can be reasonably approximated by

$$
K_{v}(x)=\frac{\Gamma(|v|)}{2}\left(\frac{2}{x}\right)^{|v|}, \quad v \neq 0 .
$$

Capitalizing on (25), taking the first term of the summation over $k$, i.e., $k=0$, which demands for $c_{0}=\left(1 / m_{1}\right)^{N-1}$, and after performing long mathematical manipulations. Thus, the asymptotic PDF and CDF can be attained, respectively, as

$$
\begin{aligned}
& f_{X}(x)=\frac{\left(1 / m_{1}\right)^{N-1} \Gamma\left(\left|m_{2} N-m_{1} N\right|\right)}{\left[\Gamma\left(m_{1}\right)\right]^{N} \Gamma\left(m_{2} N\right) N^{m_{1} N-1}} \\
& \times\left(\frac{m_{1} m_{2} N D^{\alpha}}{K_{1} \Omega_{2}}\right)^{\zeta} \sum_{\ell=1}^{n} \frac{B_{\ell}}{\left(\beta_{\ell}+\alpha \zeta+1\right)} x^{\zeta-1},
\end{aligned}
$$

and

$$
\begin{aligned}
& F_{X}(x)=\frac{\left(1 / m_{1}\right)^{N-1} \Gamma\left(\left|m_{2} N-m_{1} N\right|\right)}{\zeta\left[\Gamma\left(m_{1}\right)\right]^{N} \Gamma\left(m_{2} N\right) N^{m_{1} N-1}} \\
& \times\left(\frac{m_{1} m_{2} N D^{\alpha}}{K_{1} \Omega_{2}}\right)^{\zeta} \sum_{\ell=1}^{n} \frac{B_{\ell}}{\left(\beta_{\ell}+\alpha \zeta+1\right)} x^{\zeta},
\end{aligned}
$$

where $\zeta=\min \left\{m_{1} N, m_{2} N\right\}$.

Outage Probability: The asymptotic outage probability with aid of (27) can be written as

$$
\begin{aligned}
P_{\text {out }} & =F_{X}\left(x_{0}\right)=\frac{\left(1 / m_{1}\right)^{N-1} \Gamma\left(\left|m_{2} N-m_{1} N\right|\right)}{\zeta\left[\Gamma\left(m_{1}\right)\right]^{N} \Gamma\left(m_{2} N\right) N^{m_{1} N-1}} \\
& \times\left(\frac{m_{1} m_{2} N D^{\alpha}}{K_{1} \Omega_{2}}\right)^{\zeta} \sum_{\ell=1}^{n} \frac{B_{\ell}}{\left(\beta_{\ell}+\alpha \zeta+1\right)} x_{0}^{\zeta} .
\end{aligned}
$$

Note that the diversity order of (28) is $\zeta=$ $\min \left\{m_{1} N, m_{2} N\right\}$.

\section{Simulation Results and Discussions}

In this Section, representative numerical examples are presented to understand the impact of various parameters on the system performance. Also, Monte Carlo simulations are provided to corroborate the presented 


$$
\begin{aligned}
C=\frac{N}{\alpha \ln (2)\left[\Gamma\left(m_{1}\right)\right]^{N} \Gamma\left(m_{2} N\right)} \sum_{k=0}^{\infty} \sum_{\ell=1}^{n} c_{k} \frac{B_{\ell}}{D^{\beta_{\ell}+1}}\left(\frac{K_{1} \Omega_{2}}{m_{1} m_{2} N}\right)^{\frac{\beta_{\ell}+1}{\alpha}}\left(\frac{1}{N}\right)^{k+m_{1} N} \\
\times \mathrm{G}_{3,5}^{4,2}\left[\left.\frac{m_{1} m_{2} N D^{\alpha}}{K_{1} \Omega_{2}}\right|_{m_{2} N+\frac{\beta_{\ell}+1}{\alpha}, k+m_{1} N+\frac{\beta_{\ell}+1}{\alpha}, \frac{\beta_{\ell}+1}{\alpha}, \frac{\beta_{\ell}+1}{\alpha}, 0}, 1+.\right.
\end{aligned}
$$

$$
\begin{aligned}
\rho_{T o l}=\frac{N(1-\tau)}{\alpha \ln (2)\left[\Gamma\left(m_{1}\right)\right]^{N} \Gamma\left(m_{2} N\right)} \sum_{k=0}^{\infty} \sum_{\ell=1}^{n} c_{k} \frac{B_{\ell}}{D^{\beta_{\ell}+1}}\left(\frac{K_{1} \Omega_{2}}{m_{1} m_{2} N}\right)^{\frac{\beta_{\ell}+1}{\alpha}}\left(\frac{1}{N}\right)^{k+m_{1} N} \\
\quad \times \mathrm{G}_{3,5}^{4,2}\left[\left.\frac{m_{1} m_{2} N D^{\alpha}}{K_{1} \Omega_{2}}\right|_{m_{2} N+\frac{\beta_{\ell}+1}{\alpha}, k+m_{1} N+\frac{\beta_{\ell}+1}{\alpha}, \frac{\beta_{\ell}+1}{\alpha}, \frac{\beta_{\ell}+1}{\alpha}, 0}, 1+.\right.
\end{aligned}
$$

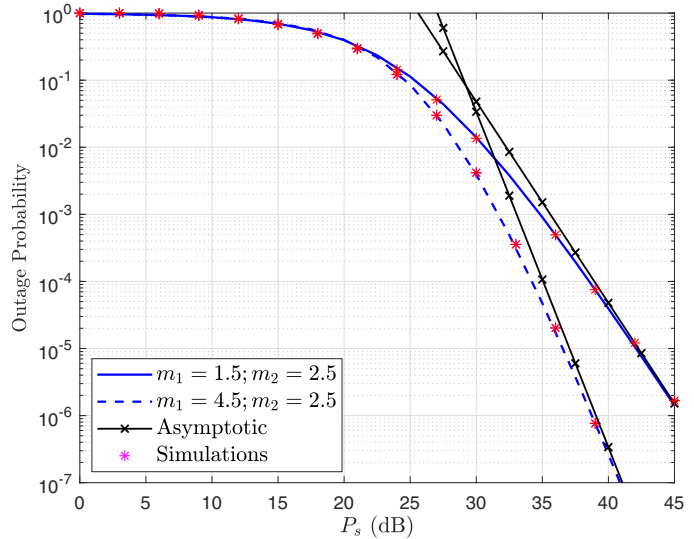

Fig. 2: Outage probability vs. $P_{t}$ for $2 \mathrm{D}$ topology with $\tau=0.6$ and $N=2$.

analysis. In all results, unless otherwise stated, the pathloss exponent is set to 2.5 while the maximum distance between the AP and MU is set to $10 \mathrm{~m}$. Also, the energy conversion efficiency $\lambda$ and the transmission rate $R$ are set to 0.75 and 2 , respectively. In addition, $D_{2}$ is set to $1 \mathrm{~m}$.

The outage probability performance as a function of $P_{s}$ for 2D topology, with $\tau=0.6$ and $N=2$, is plotted in Fig. 2. As expected, the outage probability performance improves as $P_{s}$ increases. The results also show that increasing $m_{1}$ leads to a significant outage probability performance improvement and especially a higher diversity order as reflected by the steeper slope of the corresponding outage probability curves in the high $P_{s}$ regime. For example, when $m_{1}=1.5$ and $m_{2}=2.5$, the diversity order is $\zeta=\min \left\{m_{1} N, m_{2} N\right\}$ and equals to 3 . However, when $m_{1}$ increases from 1.5 to 4.5 , the diversity order increases from 3 to 5 , which reflects the steeper slope of the corresponding outage probability curves.

In Fig. 3, the average throughput of delay-limited transmission mode versus the time-splitting ratio $\tau$ and various values of $P_{s}$ with $N=2$ is depicted. The

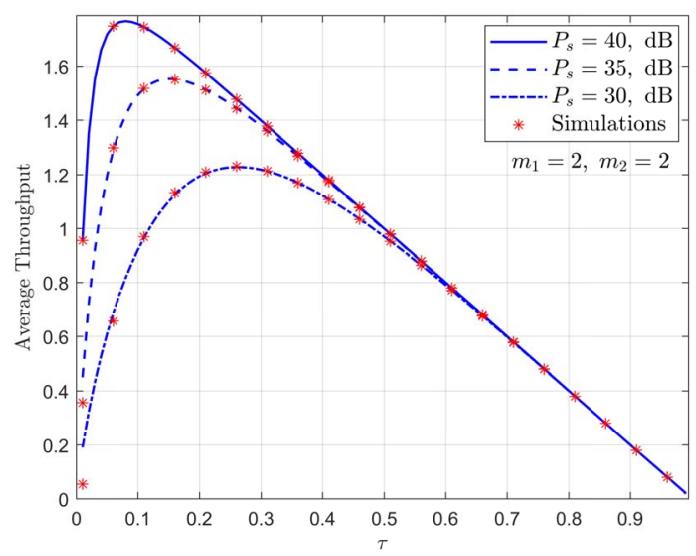

Fig. 3: Average throughput vs. $\tau$ for delay-limited transmission mode for 1D topology with $N=2$.

results show that as $P_{s}$ increases, the average throughput improves. This can be explained by the following reason. As $P_{s}$ increases, the received power at MU increases which results in increasing the average throughput. Additionally, the results show that as $\tau$ increases, the average throughput improves. However, as $\tau$ continues to increase, the average throughput reaches its maximum at some value of $\tau$, say $\tau^{*}$. As $\tau$ goes beyond $\tau^{*}$, the average throughput degrades. Also, the average throughput becomes independent of the value of $P_{s}$ as $\tau$ increases further; since the average throughput is limited by the value of $R(1-\tau)$.

The performance of average throughput for delaytolerant transmission mode against $\tau$ in 2D topology is shown in Fig. 4 for various numbers of antennas at AP with $P_{s}=25 \mathrm{~dB}$. As $N$ increases, the average throughput improves, as expected. Also, it improves as $\tau$ increases. However, as $\tau$ exceeds some values (say $\tau^{*}$ ), the average throughput starts to degrades until it reaches its minimum value as $\tau \rightarrow 1$. This because that time $\tau$ (assuming $T=1$ ) is dedicated for power transfer and 


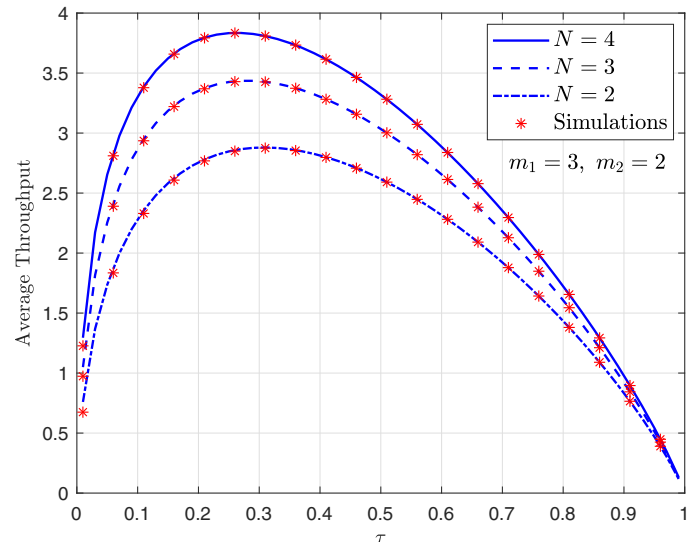

Fig. 4: Average throughput for delay-tolerant transmission mode vs. $\tau$ in $2 \mathrm{D}$ topology with $P_{s}=25 \mathrm{~dB}$.

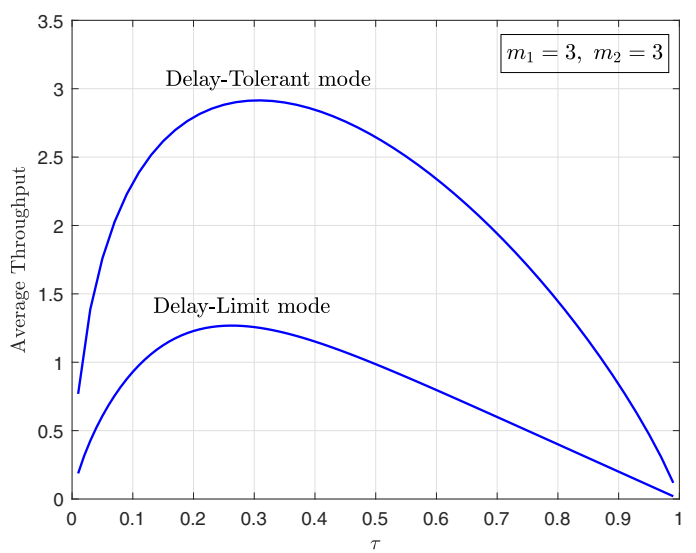

Fig. 5: Average throughput for delay-tolerant and delaylimited transmission modes in 2D topology with $P_{s}=$ $30 \mathrm{~dB}, N=2$.

the of time, i.e., $1-\tau$, is used for data transmission. For $\tau<\tau^{*}$, less time is used for power transfer, while much time is used for data transmission, thus the average throughput improves. However, as $\tau>\tau^{*}$, the time dedicated for data transmission, compared with that of power transfer, becomes inadequate to achieve better throughput.

Fig. 5 depicts the average throughput of delay-limit and delay-tolerant transmission modes in 2D topology. We can see from Fig. 5 that the delay-tolerant transmission mode achieves better performance when compared of that of delay-limit transmission mode. This is because the average throughput in the delay-tolerant transmission mode is limited by $(1-\tau) C$, while in the delay-limit transmission mode it is limited by $(1-\tau) R$. Also, in Fig. 5, we can quantify the optimal value of $\tau$ which maximizes the throughput. For the delay-tolerant mode, the optimal value of $\tau$ is about 0.3 . On the other hand, for the delay-limit mode, the optimal values of $\tau$ is about 0.25 .

\section{REFERENCES}

[1] A. A. Nasir, X. Zhou, S. Durrani, and R. A. Kennedy, "Relaying protocols for wireless energy harvesting and information processing," IEEE Trans. Wireless Commun., vol. 12, no. 7, pp. 36223636, July 2013.

[2] Y. Alsaba, S. K. A. Rahim, and C. Y. Leow, "Beamforming in wireless energy harvesting communications systems: A survey," IEEE Commun. Surv. Tut., vol. 20, no. 2, pp. 1329-1360, Jan. 2018.

[3] X. Chen, Z. Zhang, H. Chen, and H. Zhang, "Enhancing wireless information and power transfer by exploiting multi-antenna techniques," IEEE Commun. Mag., vol. 53, no. 4, pp. 133-141, Apr. 2015.

[4] L. Liu, R. Zhang, and K. Chua, "Multi-antenna wireless powered communication with energy beamforming," IEEE Trans. Commun., vol. 62, no. 12, pp. 4349-4361, Dec 2014.

[5] M. Gregori and M. Payaró, "Optimal power allocation for a wireless multi-antenna energy harvesting node with arbitrary input distribution," in IEEE ICC, Ottawa, ON, Canada, Jun. 2012, pp. 5794-5798.

[6] X. Jiang, C. Zhong, Z. Zhang, and G. K. Karagiannidis, "Power beacon assisted wiretap channels with jamming," IEEE Trans. Wireless Commun., vol. 15, no. 12, pp. 8353-8367, Dec. 2016.

[7] K. Huang and V. K. N. Lau, "Enabling wireless power transfer in cellular networks: Architecture, modeling and deployment," IEEE Trans. Wireless Commun., vol. 13, no. 2, pp. 902-912, Feb. 2014

[8] C. Zhong, X. Chen, Z. Zhang, and G. K. Karagiannidis, "Wireless-powered communications: Performance analysis and optimization," IEEE Trans. Commun., vol. 63, no. 12, pp. 51785190, Dec. 2015.

[9] H. Ju and R. Zhang, "Throughput maximization in wireless powered communication networks," in IEEE GlobeCom, Atlanta, GA, USA, Dec. 2013, pp. 4086-4091.

[10] F. Zhao, H. Lin, C. Zhong, Z. Hadzi-Velkov, G. K. Karagiannidis, and Z. Zhang, "On the capacity of wireless powered communication systems over Rician fading channels," IEEE Trans. Commun., vol. 66, no. 1, pp. 404-417, Jan. 2018.

[11] W. Huang, H. Chen, Y. Li, and B. Vucetic, "On the performance of multi-antenna wireless-powered communications with energy beamforming," IEEE Trans. Veh. Technol., vol. 65, no. 3, pp. 1801-1808, Mar. 2016.

[12] Q. Gu, G. Wang, R. Fan, Z. Zhong, K. Yang, and H. Jiang, "Rateenergy tradeoff in simultaneous wireless information and power transfer over fading channels with uncertain distribution," IEEE Trans. Veh. Technol., vol. 67, no. 4, pp. 3663-3668, Apr. 2018.

[13] Y. Chen, D. B. da Costa, and H. Ding, "Effect of CCI on WPC with time-division energy and information transmission," IEEE Wireless Commun. Lett., vol. 5, no. 2, pp. 168-171, Apr. 2016.

[14] O. S. Badarneh, "Space-shift-keying-based wireless-powered communications," IEEE Wireless Commun. Lett., vol. 7, no. 4, pp. 670-673, Aug. 2018.

[15] R. Morsi, D. S. Michalopoulos, and R. Schober, "Multiuser scheduling schemes for simultaneous wireless information and power transfer over fading channels," IEEE Trans. Wireless Commun., vol. 14, no. 4, pp. 1967-1982, Apr. 2015.

[16] L. Liu, R. Zhang, and K. Chua, "Wireless information and power transfer: A dynamic power splitting approach," IEEE Trans. Commun., vol. 61, no. 9, pp. 3990-4001, Sep. 2013.

[17] K. Govindan, K. Zeng, and P. Mohapatra, "Probability density of the received power in mobile networks," IEEE Trans. Wireless Commun., vol. 10, no. 11, pp. 3613-3619, Nov. 2011.

[18] I. S. Gradshteyn and I. M. Ryzhik, Table of Integrals, Series, and Products, 7th ed. Academic Press, California, 2007.

[19] V. S. Adamchik and O. I. Marichev, "The algorithm for calculating integrals of hypergeometric type functions and its realization in reduce system," in Proc. Intern. Conf. on Symbolic and Algebraic Computation, 1990, pp. 212-224.

[20] A. P. Prudnikov, Y. A. Brychkov, and O. I. Marichev, Integrals and Series: More Special Functions. Gordon \& Breach Sci. Publ., New York, 1990, vol. 3. 